\title{
Estrategia didáctica mediada por memes para el fortalecimiento de la lectura crítica
}

\section{Didactics strategy mediated by memes to strengthen critical reading}

\author{
Yudy Tatiana Torres Bernal ${ }^{1}$ \\ Universidad Pedagógica y Tecnológica de \\ Colombia, Duitama, Colombia. \\ Jorge Armando Niño Vega ${ }^{2}$ \\ Universidad Pedagógica y Tecnológica de \\ Colombia, Duitama, Colombia.
}

\section{RESUMEN}

Este artículo reporta los hallazgos de un estudio que tuvo por objeto fortalecer la competencia de lectura crítica a estudiantes de básica secundaria, a través una estrategia didáctica basada en memes. El trabajo se realizó bajo un enfoque mixto de alcance descriptivo y de tipo

$1 \quad$ Docente, Estudiante de Maestría en TIC Aplicadas a las Ciencias de la Educación, Especialista en Pedagogía de los Derechos Humanos, Licenciada en Idiomas Modernos Español- inglés. Universidad Pedagógica y Tecnológica de Colombia, Duitama, Colombia. E-mail: yudytatiana.torres@uptc.edu.co | ORCID ID: https://orcid.org/00000002-0017-7418

2 Docente Investigador, Magister en TIC Aplicadas a las Ciencias de la Educación, Licenciado en Tecnología. Universidad Pedagógica y Tecnológica de Colombia, Duitama, Colombia. E-mail: Jorge.ninovega@gmail.com | ORCID ID: https://orcid.org/0000-0001-7803-5535 investigación acción. La muestra correspondió a 31 estudiantes de grado noveno pertenecientes a una institución educativa colombiana. EI diagnóstico inicial indica que la mayoría de los estudiantes no dedica tiempo a la lectura y, por ende, no logra reconocer las ideas principales y secundarias de un texto. Para la intervención pedagógica se llevaron a cabo 7 escenarios, en donde se realizaron actividades acordes a las necesidades de los estudiantes. Luego del trabajo en el aula, los estudiantes lograron adquirir habilidades en relación a la lectura crítica. Se concluye que la estrategia didáctica mediada por memes es adecuada para fortalecer la lectura crítica, luego del incremento del número de estudiantes que superaron las 
pruebas inicial y final de un $18 \%$ a un $82 \%$; al igual que el nivel de aceptación que tuvieron los estudiantes por la estrategia didáctica, ya que les fue innovadora y aumentó su disposición por aprender.

PALABRAS CLAVE: Estrategia didáctica, lectura crítica, educación, TIC, memes.

\section{ABSTRACT}

This article reports the findings of a study which aimed to strengthen the critical secondary reading competence of elementary school students, through a didactic strategy based on memes. The work was carried out under a mixed approach of descriptive scope and action research type. The sample corresponded to 31 ninth-grade students from a Colombian educational institution. The initial diagnosis indicates that most students do not spend time reading and, therefore, fail to recognize the main and supporting ideas of a text. For the pedagogical intervention, 7 environments were carried out, where activities were carried out according to the needs of the students. After working in the classroom, the students were able to acquire skills in relation to critical reading. It is concluded that the didactic strategy mediated by memes is adequate to strengthen critical reading, after the increase in the number of students who passed the initial and final tests from $18 \%$ to $82 \%$; as well as the level of acceptance that students had for the didactic strategy, since it was innovative and increased their willingness to learn.

KEYWORDS: Didactic strategy, critical reading, education, ICT, memes.

\section{INTRODUCCIÓN}

Con el pasar del tiempo y gracias a los avances científicos y tecnológicos hechos por el hombre, el tener acceso a dispositivos tecnológicos y ha conexión a internet ahora es más fácil gracias a la producción en masa tanto de artefactos tecnológicos, así como de aplicaciones digitales
(Orozco-Báez et al., 2020; Avella-lbáñez et al., 2017). El acceso a esta tecnología es producto de la globalización, lo que ha generado impactos favorables para varios sectores, tales como: la educación, la política, la cultura, el deporte, etc. (Suárez-Triana et al., 2020; Niño-Vega et al., 2020; Padilla-Ospina et al., 2020).

No obstante, la tecnología también ha tenido impactos desfavorables debido al mal uso que le dan algunas personas, lo cual las convierte en consumidoras y dependientes de herramientas tecnológicas y digitales (QuitianFeliciano et al.,2020; Díaz-Garay et al., 2020; Niño vega et al., 2016). Una consecuencia que ha traído consigo la dependencia tecnológica, es la perdida de interés por parte de los niños $y$ adolescentes en cuanto a realizar actividades físicas y mentales, las cuales les contribuya en su desarrollo integro y emocional (Cruz-Rojas et al., 2019; Garzón-Saladen \& Romero-González, 2018; Gutiérrez-Rico et al., 2019; Niño-Vega et al., 2017).

En la actualidad, se puede evidenciar que los estudiantes dedican gran parte de su tiempo, en realizar actividades como: jugar videojuegos, chatear con amigos, navegar en internet y en redes sociales y visitar plataformas de contenido audiovisual (Espinel-Rubio et al., 2020; NiñoVega et al., 2020; Ducuara-Amado et al., 2020; Naranjo-Gaviria \& Herreño-Téllez, 2020). Esto ha ocasionado que los jóvenes, dediquen poco tiempo a sus estudios y por tal razón, su rendimiento académico disminuya (ClavijoCáceres, 2020; Castro-Robles et al., 2020; NiñoVega et al., 2019; Galeano-Becerra et al., 2018).

Uncaso particulardel bajo rendimientoacadémico se evidencia en el área de humanidades, ya que los estudiantes dedican poco tiempo a la lectura y por tal razón, no adquieren las competencias necesarias para comprender la información que se presenta en un texto (Bernate et al., 2020; Borjas et al., 2019; Alvis-Puentes et al., 2019). Este caso se evidencia con estudiantes de 
noveno grado pertenecientes al Instituto Técnico industrial Rafael Reyes ubicado en la ciudad de Duitama-Boyacá. Los cuales, en los simulacros realizados en la institución educativa, se muestra que el desempeño en lectura crítica se encuentra por debajo de los 45 puntos de 100 .

A lo expuesto, se identificó que la falencia que presentan los estudiantes de dicha institución educativa, es lograr abstraer ideas principales y secundarias de textos expositivos y descriptivos. Por tal razón, en esta investigación se propuso emplear algunas herramientas tecnológicas $\mathrm{y}$ digitales con el fin de desarrollar una estrategia didáctica basada en memes, el cual permita fortalecer las competencias en lectura crítica de los estudiantes. A continuación, se detalla la metodología que se siguió para la ejecución de este estudio. Igualmente, se presentan los resultados obtenidos en las 3 fases propuestas para el diseño, desarrollo y validación de la estrategia didáctica.

\section{METODOLOGÍA}

El presente estudio se realizó desde un enfoque mixto, debido a que se empleó el enfoque cuantitativo para identificar en base a la medición numérica, los conocimientos de cada uno de los estudiantes, antes y después de haber sido intervenidos con la estrategia didáctica propuesta. De igual forma se empleó el enfoque cualitativo, con el fin de conocer los hábitos de lectura que tiene cada estudiante, así como su percepción en cuanto a la estrategia didáctica empleada (Hernández-Sampieri et al., 2010).

Por otra parte, la investigación es de alcance descriptivo y de tipo investigación Acción, debido a que se estudió el impacto de una estrategia didáctica basada en memes para fortalecer la lectura crítica de los estudiantes.

En cuanto a la población objeto de estudio, se trabajó con 31 estudiantes de grado noveno pertenecientes a la institución educativa, Instituto Técnico Industrial Rafael Reyes, ubicado en la ciudad de Duitama-Boyacá. La intervención se efectuó durante el primer y segundo periodo académico del año 2020.

Como instrumentos de medida para esta investigación, se emplearon 2 encuestas y 2 cuestionarios. La primera encuesta se empleó para identificar los hábitos de los estudiantes en cuanto a dedicación a la lectura, el acceso a redes sociales y el uso de memes. La segunda encuesta se empleó para obtener la percepción de los estudiantes frente a la estrategia pedagógica propuesta. En cuanto a los 2 cuestionarios, uno de ellos fue empleado al inicio del estudio con el fin de identificar los conocimientos y habilidades que traían los estudiantes en cuanto a la lectura crítica, capacidad de síntesis y abstracción de una idea central de un tema. De igual forma, el segundo cuestionario se empleó para identificar los conocimientos y habilidades que obtuvieron los estudiantes entorno a la lectura crítica, luego de haber sido intervenidos con la estrategia pedagógica desarrollada.

La investigación se desarrolló en tres fases, en donde la fase 1 se realizó una caracterización de los hábitos de lectura y uso de los memes por parte de los estudiantes, con el fin de identificar las temáticas a orientar a través de la estrategia.

En la fase 2 se propuso la estrategia pedagógica a desarrollar, en esta se establecieron los escenarios, las actividades de aprendizaje, la forma en cómo se iba a medir los conocimientos de los estudiantes, el tiempo de ejecución de la actividad, así como el modelo pedagógico a emplear durante la intervención en el aula de clases.

En cuanto a la fase 3, se efectuó la validación de la estrategia didáctica; para ello se realizó la orientación pedagógica acorde a la estrategia didáctica propuesta y finalmente se identificó su nivel de impacto a través de los desempeños 
obtenidos en el cuestionario final en relación al cuestionario inicial. Igualmente, se validó el nivel de aceptabilidad que tuvo la estrategia didáctica a través de la opinión de cada estudiante.

\section{RESULTADOS Y DISCUSIÓN}

\subsection{Caracterización de hábitos de lectura y uso de memes}

En primera medida, se realizó una encuesta a los 31 estudiantes que participaron en esta investigación a través de la herramienta digital "Google Forms". En esta encuesta se les preguntó a los estudiantes ¿Cuánto tiempo dedica diariamente a la lectura?; a ello, 19 estudiantes manifestaron no dedicar tiempo a la lectura, 9 estudiantes indicaron dedicar 1 hora diaria y 3 estudiantes manifestaron hacer más de 2 horas de lectura al día.

En cuanto al tipo de lectura que más les llama la atención, 10 de los 12 estudiantes que, si dedican tiempo a la lectura, indicaron que prefieren textos que narren sobre sucesos históricos o actuales que acontecen alrededor del mundo. En cuanto a los 2 estudiantes restantes, prefieren leer comics o mangas.

Con respecto al medio que prefieren que los estudiantes se les presenten las lecturas, los 31 estudiantes coincidieron en que prefieren de manera digital, ya que pueden acceder a estas desde cualquier dispositivo tecnológico, así como compartir con cualquier otra persona, del mismo modo, es más ecológico al no utilizar papel.

Posteriormente, se les preguntó a los estudiantes si reconocen que es un meme y para que se utiliza, ante esta pregunta todos los estudiantes manifestaron conocer que era un meme e indicaron que los memes son una expresión gráfica, en el cual ya sea por medio de una figura, una imagen o una ilustración, se relata la vida cotidiana, la cultura, los comportamientos, las emociones de las personas de manera graciosa.

A ello, a los estudiantes se les indagó si utilizaban memes para expresarse; ante esto, los 31 estudiantes indicaron que sí, de la siguiente forma: 14 de ellos indicaron utilizar la tipología del meme "celebridades", 7 por el estilo "Rage Guys Troll Face", 4 estudiantes por "animales", 3 estudiantes en cuanto "marketing", y 3 estudiantes emplean memes de tipos "animados". De igual forma, se identificó que 13 estudiantes hacen sus propios memes, 12 estudiantes los copian y los 6 estudiantes restantes los editan. En cuanto a las temáticas que les gusta ver en los memes a los estudiantes son: humor, política, cultura y académicos.

Por otra parte, con el fin de identificar las redes sociales las cuales emplean los estudiantes para compartir memes, se denoto que Facebook es utilizada por 23 estudiantes, WhatsApp por 5 estudiantes e Instagram por 3 estudiantes.

\subsection{Estrategia didáctica propuesta}

Una vez identificados los hábitos de lectura que tienen los estudiantes, los tipos de memes que usan las temáticas que les gusta ver y compartir, así como las redes sociales más utilizadas por parte de ellos, se procedió a establecer una estrategia pedagógica, con el fin de fomentar la lectura crítica y su representación gráfica de ideas a través de memes. 


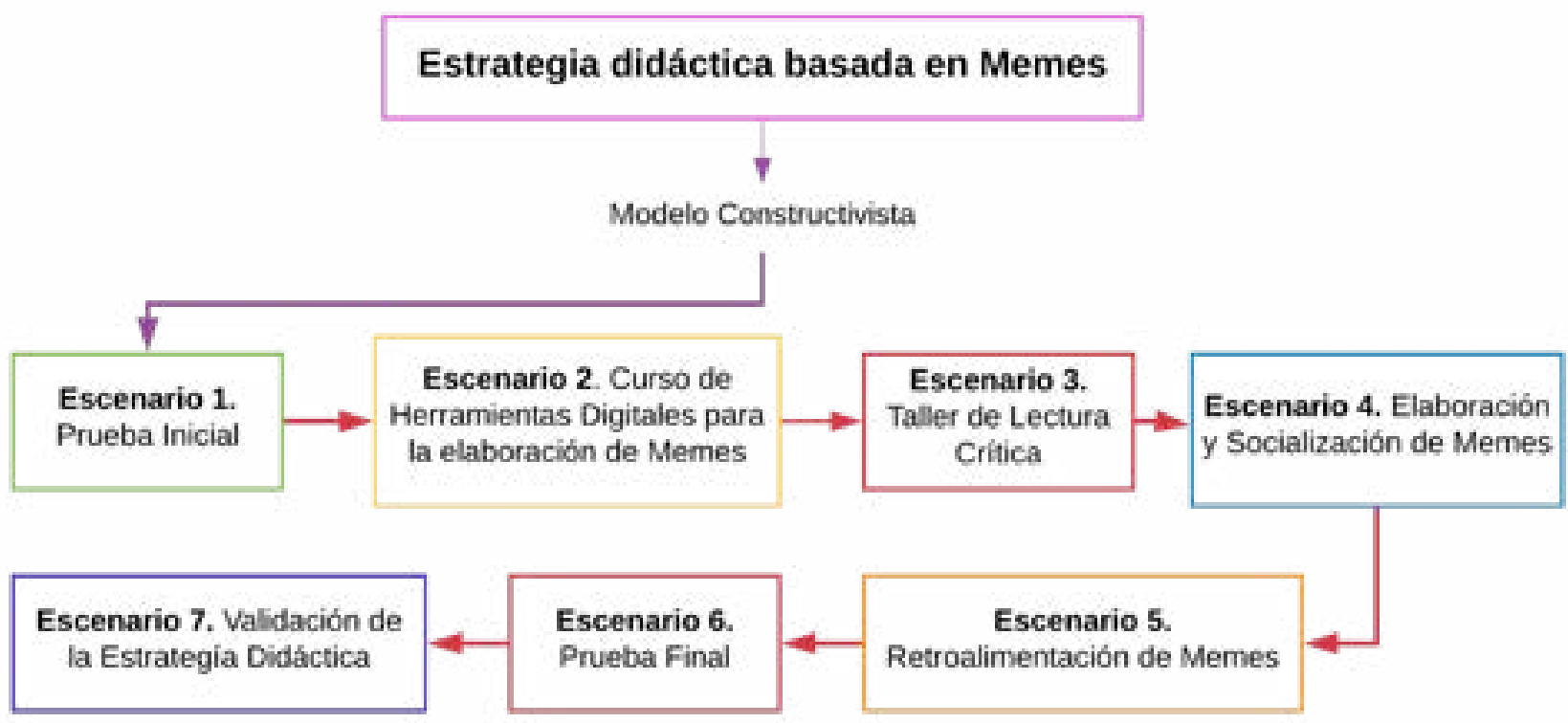

Figura 1. Estrategia didáctica propuesta

En la figura 1, se evidencia la estrategia propuesta a ser implementada para el desarrollo de la competencia de lectura crítica con la población objeto de estudio. En esta estrategia se evidencia que el modelo pedagógico a emplear es el constructivismo, en donde a través del aprendizaje colaborativo, el estudiante logrará aprender a abstraer ideas principales de un texto; en este caso como teoría didáctica se planteó el análisis del discurso. En la propuesta se plantean 5 escenarios para desarrollar en un periodo académico, correspondiente a 10 semanas de clases con una intensidad de 4 horas semanales.

En el escenario 1 se propone una prueba inicial. En esta actividad se les aplica un cuestionario inicial a los estudiantes, en donde se les mide la capacidad de síntesis y la habilidad para abstraer ideas primerias y secundarias entorno a una noticia.

En el escenario 2, se hace un curso de herramientas tecnológicas para la elaboración de memes. Para ello se enseña a los estudiantes a manejar las herramientas: Memegenerator, Photoshop Express Editor, imgflip, Meme Dad, Quickmeme, Livememe e igualmente también se orienta sobre el uso de la herramienta PowerPoint para la elaboración de los mismos. Estas herramientas tecnológicas pueden ser ejecutadas ya sea desde un computador con sistema Windows o desde un teléfono celular con sistema Android o IOS.

En el escenario 3 se plantea un taller de lectura crítica a los estudiantes. En esta actividad se les brinda algunas noticias de actualidad tomadas de la revista Semana y de periódicos como el Espectador y el Tiempo. A ello, se les orienta a los estudiantes sobre como abstraer las ideas centrales de una noticia, e igualmente se les brinda tips, los cuales le ayudan a reforzar el pensamiento crítico del estudiante frente a la temática que se les presentan en la noticia.

En el escenario 4 se estable la elaboración y socialización de memes en relación a las lecturas realizadas. Para ello, los estudiantes elaboran uno o más memes empleando alguna de las herramientas tecnológicas vistas en el curso. Cómo aspecto a resaltar, el meme debe expresar la opinión del estudiante frente a la idea central reportada en la noticia. De igual manera, los estudiantes deben socializar su meme elaborado con sus compañeros de clases, a 
través de la red social Facebook y WhatsApp.

En el escenario 5 se planteó la retroalimentación de los memes. En esta actividad, los estudiantes deben realizar sus comentarios frente a los memes elaborados de sus compañeros. Si fuera el caso, el autor del meme debía explicar la intención del mismo y reelaborarlo si no es entendible por los demás estudiantes.

En el escenario 6 se estableció la prueba final. Con esta actividad lo que se espera es identificar las habilidades que adquirieron los estudiantes en cuanto a las competencias en lectura crítica. Para ello se realiza una prueba similar a la prueba inicial y adicionalmente se les pide a los estudiantes que debatan con argumentos su posición neutral, a favor o en contra de la noticia que le corresponde.

Finalmente, en el escenario 7 se hace la validación de la estrategia didáctica, para ello se aplica una encuesta de satisfacción con el fin de conocer la percepción que tiene cada estudiante frente a la estrategia implementada y de ser posible se realizan mejoras ya sea en los contenidos o en la forma de explicar los mismos.

\subsection{Validación de la estrategia didáctica}

En relación al diseño de la estrategia didáctica propuesta, se realizó la ejecución de cada uno de los 7 escenarios establecidos. Para ello, se dispuso de 40 horas de intervención con los estudiantes, dividido en lo que corresponde a las 10 semanas de clases con las que se contó para el primer y parte del segundo periodo académico del año 2020.

En el pretest propuesto en el escenario 1, se evidenció que tan solo 2 de los 31 estudiantes saben abstraer la idea central de una notica de opinión pública. De igual forma, 5 estudiantes lograron plasmar correctamente su punto de vista en relación con la temática que se presentó en la noticia, Por otra parte, 10 estudiantes saben interpretar las cifras que se reportan en las columnas de la Revista Semana, sin embargo, debido al poco tiempo que le dedican a la lectura, se les dificulta comprender temáticas a las que los periodistas citan.

En cuanto a la ejecución del escenario 2, se observó un gran interés por parte del estudiante al momento de aprender a manejar las diferentes herramientas tecnológicas para la elaboración de memes. Igualmente, además de enseñarle a los estudiantes a manejar estas herramientas digitales, también se les enseñó sobre la importancia de los derechos de autor, a lo cual, se les asesoro sobre banco de imágenes libres como pixabay y freepik.

Con respecto al taller de lectura propuesto en el escenario 3 , a los estudiantes se les dio un tiempo de 8 horas distribuidas en 2 semanas de clases, para que tomaran al menos 3 noticias ya sea del periódico el tiempo, el espectador o la revista semana, las analizara y extrajera la idea central y secundaria, así como compartiera su punto de vista en base a los tips orientados en clases.

En el desarrollo de las actividades de los escenarios 4 y 5 , los estudiantes plasmaron a través de memes, las ideas centrales o sus opiniones frente a la noticia que seleccionaron y las compartieron a través de la red social Facebook, en el siguiente grupo:<<https://www. facebook.com/Castellano-class-2020-TatianaTorres-102860428171590/>>. Para ello, en la figura 2 se evidencia algunos de los memes elaborados por los estudiantes, tanto en el cuaderno de clases como primer borrador, como la versión final luego de haberlos digitalizados en los softwares vistos en clases. 


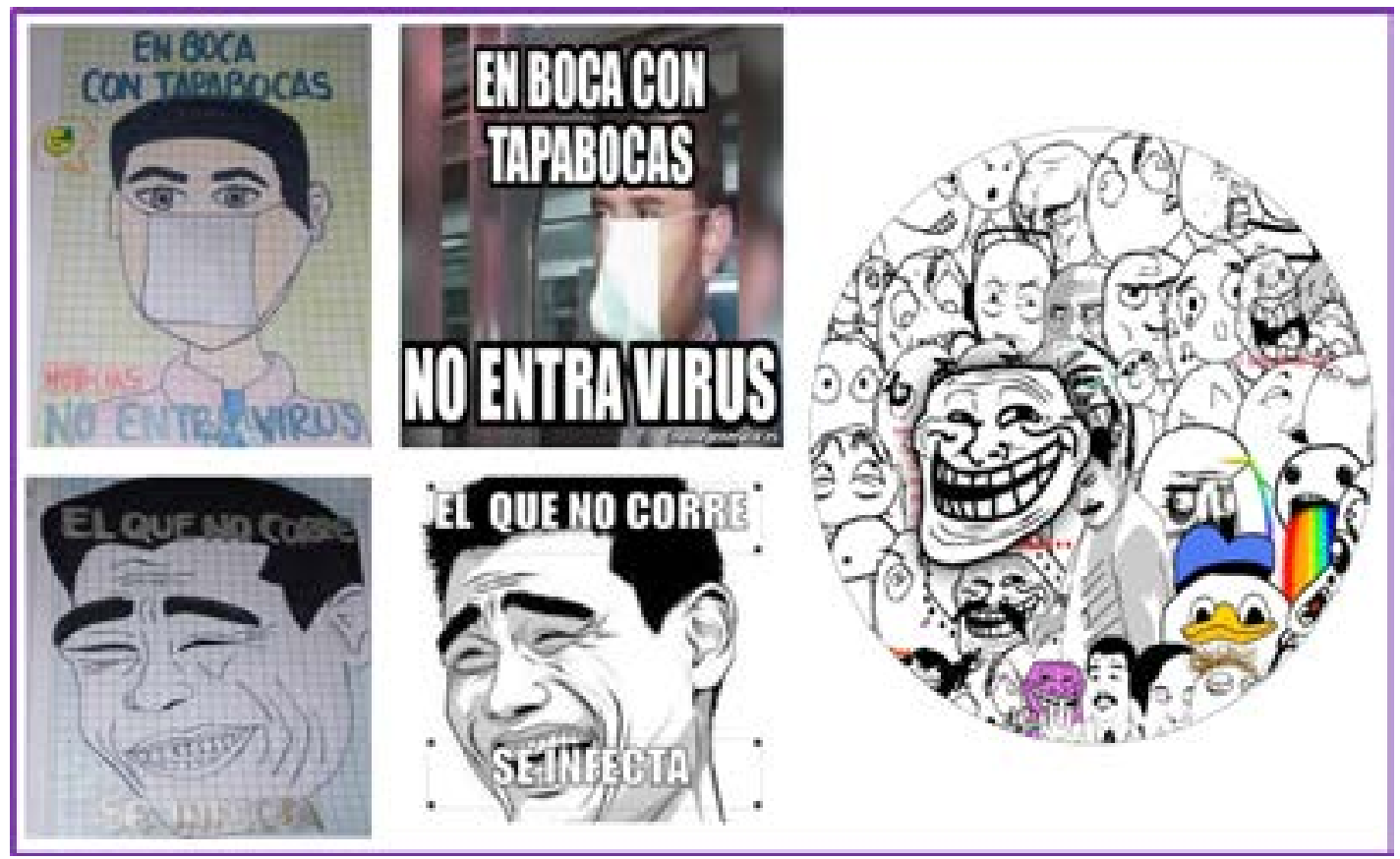

Figura 2. Representación grafica de ideas a través de memes

Por otra parte, en el escenario 6 se les realizo a los 31 estudiantes objeto de este estudio un test final, con el objetivo de identificar si la estrategia didáctica mediada por memes, tuvo efecto en cuanto al desarrollo de la competencia lectura crítica. Aello, 29 estudiantes lograron abstraer las ideas primarias y secundarias de una noticia. De igual forma, 30 estudiantes adquirieron criterios sólidos para argumentar, defender o criticar una opinión publica reportada en un artículo. Por último, 23 de los 31 estudiantes adquirieron la habilidad de interpretar información no textual, como lo son los datos estadísticos que se reportan a manera de tablas o gráficos.

En cuanto a la validación de la estrategia didáctica por parte del estudiante, propuesta en el escenario 7 , se obtuvo la siguiente información: con respecto a la pregunta ¿Cómo les pareció el curso de lectura crítica?, a ello 26 estudiantes les pareció excelente, debido a que les permitió identificar la manera correcta en cómo se debe leer un documento y como abstraer información precisa de ellos; en cuanto a los 5 estudiantes restantes les pareció buena, aunque indicaron que las noticias presentadas eran algo difíciles de comprender si solo se leía una vez el documento.

En cuanto a la pregunta ¿Qué tan agradable les pareció los memes que se realizaron entorno a una noticia?, ante este interrogante los 31 estudiantes manifestaron ser de mucho agrado, debido a que les pareció una actividad muy divertida ya que aprendieron a crear sus propios memes y lograron conocer y representar sucesos y acontecimientos que ocurren en el mundo a través de los memes.

Finalmente, con respecto a la pregunta ¿vas a dedicar más tiempo a la lectura?, 29 estudiantes manifestaron que sí, ya que les permite estar informados de los acontecimientos más importantes que pasan en el entorno. De igual manera, los estudiantes indicaron que el leer les abre panoramas que a futuro les contribuye de manera personal y profesionalmente, en cuanto a la agilidad de abstraer ideas, resumir, sintetizar y de juzgar con base en criterios solidos que se adquieren luego de haber leído hallazgos reportados por diversos investigadores. 


\subsection{DISCUSIÓN}

Los resultados de este estudio permitieron comprobar la efectividad que tuvo una estrategia didáctica basada en memes para el fortalecimiento de la lectura crítica en estudiantes de noveno grado. Lo anterior se corrobora gracias a la ganancia de aprendizaje que tuvieron los estudiantes, debido a que, en la prueba inicial, tan solo fue aprobada por 6 estudiantes $(18 \%)$, mientras que, en la prueba final aprobaron 25 estudiantes (82\%).

Lo anterior se debe gracias al diseño y la elaboración tanto de la estrategia didáctica, así como el material educativo empleado para el desarrollo de las actividades propuestas para cada escenario; esto ya que se pensó de acuerdo a las necesidades identificadas por cada estudiante. Investigaciones relacionadas, han podido establecer que la base del éxito para que el estudiante realmente aprenda, es identificar sus hábitos de estudio, reconocer las habilidades e intereses que estos tienen y abordar una temática, el cual sea de interés para los educandos y permitan reconocer su función y aplicabilidad (Hernández-Gil \& Núñez-López, 2020; Pineda-Ospina et al., 2020; HernándezGil \& Jaramillo-Gaitán,2020). De esta manera, cualquier material educativo tendrá gran acogida por parte del estudiante y logrará tener efectividad en cuanto al proceso de enseñanzaaprendizaje (Ruiz-Macías \& Duarte, 2018; Vesga-Bravo \& Escobar-Sánchez, 2018; LópezGaitán et al., 2018; Niño-Vega et al., 2019).

Por otra parte, con esta investigación se logró identificar que el uso de los memes y las redes sociales son benefactoras en el proceso de enseñanza-aprendizaje, siempre y cuando se tenga clara la estrategia en cómo se va a orientar adecuadamente al estudiante, en cuál va ser el contenido que se le va a presentar, cómo será el medio de comunicación para guiarlo y de qué forma se le va a evaluar. A ello, algunos estudios han denotado el fuerte impacto que tienen las Tecnologías de la Información y la Comunicación, TIC, en la educación, ya que permite recrear ambientes de aprendizaje virtuales que favorecen al estudiante, gracias a la disposición de varias herramientas tecnológicas, contenidos digitales y canales para la comunicación sincrónica y asincrónica con sus compañeros, docentes y expertos del área ((Vargas-Vargas et al., 2020; Fonseca-Barrera et al., 2020; Niño-Vega \& Fernández-Morales, 2019; Martínez-López \& Gualdrón-Pinto, 2018; Jiménez-Espinosa, 2019).

Es entonces que, con base a los resultados de esta investigación, así como en los hallazgos de investigaciones reportados en cuanto al desarrollo y validación de estrategias didácticas (Iriarte-Pupo, 2020; Jiménez-Espinosa \& Sánchez-Bareño, 2019). Se puede decir que: para que haya un correcto proceso de enseñanza-aprendizaje, se deben reestructurar la forma en como se está orientando los contenidos en el aula de clases, se deben pensar en la transversalidad de áreas del conocimiento y se deben optar por implementar materiales educativos y ambientes de aprendizaje actualizados (Ordóñez-Ortega et al., 2019; Martínez-Diaz \& Rodríguez-Hernández, 2020; Angarita-López et al, 2020).

\section{CONCLUSIONES}

Esta investigación tuvo la finalidad de desarrollar una estrategia didáctica basada en memes con el fin de fortalecer la competencia de lectura crítica a estudiantes de grado noveno. El diagnostico permitió identificar las falencias que tenían los estudiantes en cuanto a hábitos de lectura, capacidad de abstracción de ideas primarias y secundarias de un texto y la falta de argumentación para debatir un tema.

La intervención pedagógica que se realizó en el aula de clases, implicó la ejecución de 7 escenarios en los cuales se desarrollaron actividades de aprendizaje que permitieron a los 
estudiantes, mejorar su capacidad de síntesis y abstracción de la información de un texto, al igual que aprendieron a expresar sus opiniones a través de representaciones graficas como lo son los memes.

La estrategia didáctica tuvo gran acogida por parte de los estudiantes, ya que fue innovadora y llamativa, lo que ocasiono que los estudiantes mantuvieran el interés por aprender y desarrollar cada una de las actividades propuestas en cada escenario. De igual forma, los estudiantes aludieron que la implementación de las herramientas digitales, así como de los dispositivos tecnológicos, como recursos educativos, posibilitan un mejor ambiente de aprendizaje ya que trasciende al cotidiano.

Con esta investigación se reafirma la importancia de seguir implementado estrategias didácticas innovadoras, las cuales se familiarice con las necesidades de aprendizaje de los estudiantes y revolucionen la manera en cómo se orienta y evalúan los conocimientos que estos adquieren.

\section{REFERENCIAS BIBLIOGRÁFICAS}

Alvis-Puentes, J. F., Aldana-Bermúdez, E., \& Caicedo-Zambrano, S. J. (2019). Los ambientes de aprendizaje reales como estrategia pedagógica para el desarrollo de competencias matemáticas en estudiantes de básica secundaria. Revista de Investigación, Desarrollo e Innovación, 10(1), 135-147. https:// doi.org/10.19053/20278306.v10. $\underline{\mathrm{n} 1.2019 .10018}$

Angarita-López, R. D., Fernández-Morales, F. H., Niño-Vega, J. A., Duarte, J. E., GutiérrezBarrios, G. J. (2020). Accesibilidad de las revistas colombianas del área de humanidades bajo las pautas WCAG 2.1. Revista Espacios, 41(4), 18. http://revistaespacios.com/ a20v41n04/20410418.html
Avella-lbáñez, C. P., Sandoval-Valero, E. M., \& Montañez-Torres, C. (2017). Selección de herramientas web para la creación de actividades de aprendizaje en Cibermutua. Revista de investigación, Desarrollo e Innovación, 8(1), 107-120. https://doi.org/10.19053/20278306. v8.n1.2017.7372

Bernate, J. A., García-Celis, M. F., FonsecaFranco, I. P., \& Ramírez-Ramírez, N. E. (2020). Prácticas de enseñanza y evaluación en una facultad de educación colombiana. Revista de Investigación, Desarrollo e Innovación, 10(2), 337-347. https://doi.org/10.19053/20278306.v10. $\underline{\mathrm{n} 2.2020 .10721}$

Borjas, M. P., Navarro-Lechuga. E., PuentesOspino, D., De la cruz-García, J., Yepes-Martínez, J., Muñoz-Alvis, A., Montero, P., De La Hoz-Del Villar, K., Pérez-Moyano, Y., \& Polo, J. D. (2019). Experiencias ludoevaluativas en el contexto universitario: la evaluación desde una comunidad de aprendizaje. Revista de Investigación, Desarrollo e Innovación, 10(1), 185-199. https:// doi.org/10.19053/20278306.v10. $\underline{\mathrm{n} 1.2019 .10021}$

Castro-Robles, A., Niño-Vega, J., \& FernándezMorales, F. (2020). El acoso escolar como oportunidad para mejorar la convivencia en el aula. Educación Y Humanismo, 22(38). https://doi. org/10.17081/eduhum.22.38.3675

Clavijo-Cáceres, D. (2020). La calidad y la docencia universitaria: algunos criterios para su valoración. Revista de Investigación, Desarrollo e Innovación, 11(1).

Cruz-Rojas, G. A., Molina-Blandón, M. A., \& Valdiri-Vinasco, V. (2019). Vigilancia 
tecnológica para la innovación educativa en el uso de bases de datos y plataformas de gestión de aprendizaje en la universidad del Valle, Colombia. Revista de Investigación, Desarrollo e Innovación, 9(2), 303-317. https://doi.org/10.19053/20278306. v9.n2.2019.9175

Díaz-Garay, I. del S., Narváez-Escorcia, I. T., \& Amaya-De Armas, T. (2020). El proyecto de vida como competencia básica en la formación integral de estudiantes de educación media. Revista de Investigación, Desarrollo e Innovación, 11(1).

Ducuara-Amado, L. Y., Rodríguez-Hernández, A. A., Niño-Vega, J. A., \& FernándezMorales, F. H. (2020). Material educativo gamificado para la enseñanzaaprendizaje de conceptos de ecología en estudiantes de educación media. Revista Boletín Redipe, 9(6), 144-156. https://doi.org/10.36260/rbr.v9i6.1008

Espinel-Rubio, G. A., Hernández-Suárez, C. A., \& Rojas-Suárez, J. P. (2020). Las TIC como medio socio-relacional: un análisis descriptivo en el contexto escolar con adolescentes de educación media. Revista de Investigación, Desarrollo e Innovación, 11(1).

Fonseca-Barrera, C. C., Niño-Vega, J. A., \& Fernández-Morales, F. H. (2020). Desarrollo de competencias digitales en programación de aplicaciones móviles en estudiantes de noveno grado a través de tres estrategias pedagógicas. Revista Boletín Redipe, 9(4), 179-191. https://doi.org/10.36260/rbr.v9i4.958

Galeano-Becerra, C. J., Bellón-Monsalve, D., Zabala-Vargas, S. A., Romero-Riaño, E., \& Duro-Novoa, V. (2018). Identificación de los pilares que direccionan a una institución universitaria hacia un SmartCampus. Revista de Investigación, Desarrollo e Innovación, 9(1), 127-145. https://doi.org/10.19053/20278306. v9.n1.2018.8511

Garzón-Saladen, Á., \& Romero-González, Z. (2018). Los modelos pedagógicos y su relación con las concepciones del derecho: puntos de encuentro con la educación en derecho. Revista de Investigación, Desarrollo e Innovación, 8(2), 311-320. https:// doi.org/10.19053/20278306. v8.n2.2018.7968

Gutiérrez-Rico, D., Almaraz-Rodríguez, O. D., \& Bocanegra-Vergara, N. (2019). Concepciones del docente en sus formas de percibir el ejercicio de la investigación desde su práctica. Revista de Investigación, Desarrollo e Innovación, 10(1), 149-161. https:// doi.org/10.19053/20278306.v10. $\underline{\mathrm{n} 1.2019 .10019}$

Hernández-Gil, C., \& Jaramillo-Gaitán, F. A. (2020). Laboratorio de innovación social: hibridación creativa entre las necesidades sociales y las experiencias significativas de los estudiantes de administración de empresas. Revista de Investigación, Desarrollo e Innovación, 10(2), 267-281. https:// doi.org/10.19053/20278306.v10. $\underline{\mathrm{n} 2.2020 .10518}$

Hernández-Gil, C., \& Núñez-López, J. A. (2020) Design thinking aplicado al mejoramiento de las competencias ciudadanas en universitarios: voto popular. Revista de Investigación, Desarrollo e Innovación, 11(1). 
Hernández-Sampieri, R., Fernández-Collado, C., \& Baptista-Lucio, P. (2010). Metodología de la Investigación. Chile: McGraw Hill.

Iriarte-Pupo, A. J. (2020). Fenomenologíahermenéutica de la investigación formativa. El formador de formadores: de la imposición a la transformación. Revista de Investigación, Desarrollo e Innovación, 10(2), 311-322. https:// doi.org/10.19053/20278306.v10. n2.2020.10722

Jiménez-Espinosa, A. (2019). La dinámica de la clase de matemáticas mediada por la comunicación. Revista de Investigación, Desarrollo e Innovación, 10(1), 121-134. https://doi.org/10.19053/20278306.v10. $\underline{\mathrm{n} 1.2019 .10016}$

Jiménez-Espinosa, A., \& Sánchez-Bareño, D. M. (2019). La práctica pedagógica desde las situaciones a-didácticas en matemáticas. Revista de Investigación, Desarrollo e Innovación, 9(2), 333-346. https://doi.org/10.19053/20278306. v9.n2.2019.9179

López-Gaitán, M. A., Moran-Borbor, R. A., \& Niño-Vega, J. A. (2018). Prácticas experimentales como estrategia didáctica para la comprensión de conceptos de física mecánica en estudiantes de educación superior. Infometric@ - Serie Ingeniería, Básicas yAgrícolas, 1(1), 1-14. http://infometrica. org/index.php/syh/article/view/12

Martínez Diaz, L. Y., \& Rodríguez Hernández, A. A. (2020). El booktrailer como estrategia didáctica para el desarrollo de competencias comunicativas, narrativas y digitales. Revista Boletín Redipe, 9(6), 168-182. https://doi.org/10.36260/rbr. $\underline{\text { v9i6.1010 }}$

Martínez-López, L. G., \& Gualdrón-Pinto, E.
(2018). Fortalecimiento del pensamiento variacional a través de una intervención mediada con TIC en estudiantes de grado noveno. Revista de Investigación, Desarrollo e Innovación, 9(1), 91-102. https://doi.org/10.19053/20278306. v9.n1.2018.8156

Naranjo-Gaviria, A. A., \& Herreño-Téllez, E. (2020). Caracterización de las actividades interdisciplinarias en una facultad de artes Colombiana. Revista de Investigación, Desarrollo e Innovación, 10(2), 283-296. https:// doi.org/10.19053/20278306.v10. $\underline{\mathrm{n} 2.2020 .10361}$

Niño-Vega, J. A., \& Fernández-Morales, F. H. (2019). Una mirada a la enseñanza de conceptos científicos y tecnológicos a través del material didáctico utilizado. Revista Espacios, 40(15). http://www.revistaespacios.com/ a19v40n15/19401504.html

Niño-Vega, J. A., Fernández-Morales, F. H., \& Duarte, J. E. (2019). Diseño de un recurso educativo digital para fomentar el uso racional de la energía eléctrica en comunidades rurales. Saber, Ciencia y Libertad, 14(2), 256-272. https://doi.org/10.18041/2382-3240/ saber.2019v14n2.5889

Niño-Vega, J. A., López-Sandoval, D. P., Mora-Mariño, E. F., Torres-Cuy, M. A., \& Fernández-Morales, F. H. (2020). Método Singapur aplicado a la enseñanza de operaciones básicas con números fraccionarios en estudiantes de grado octavo. Pensamiento Y Acción, (29), 21-39. https://revistas.uptc.edu.co/ index.php/pensamiento_accion/article/ view/11270 
Niño-Vega, J. A., Martínez-Díaz, L. Y., \& Fernández-Morales, F. H. (2016). Mano robótica como alternativa para la enseñanza de conceptos de programación en Arduino. Revista Colombiana de Tecnologías de Avanzada, 2(28), 132-139. https:// doi.org/10.24054/16927257.v28. $\underline{\mathrm{n} 28.2016 .2476}$

Niño-Vega, J.A., Martínez-Díaz, L. Y., FernándezMorales, F. H., Duarte, J. E., ReyesCaballero, F., \& Gutiérrez-Barrios, G. J. (2017). Entorno de aprendizaje para la enseñanza de programación en Arduino mediado por una mano robótica didáctica. Revista Espacios, 38(60), 23. http://www.revistaespacios.com/ a17v38n60/17386023.html

Niño-Vega, J. A., Moran-Borbor, R.A., \& Fernández- Morales, F. H. (2019). Educación inclusiva: Un nuevo reto para la labor docente en el siglo XXI. Infometric@-Serie Sociales y Humanas, 1(2), 74-94. http://infometrica.org/index. php/ssh/article/view/78

Niño-Vega, J. A., Orozco-Báez, M. Y., \& Fernández-Morales, Flavio, H. (2020). El clima familiar y su relación con el fenómeno del ciberacoso en una institución educativa colombiana. Revista Espacios, 41(26), 308-317. https://www.revistaespacios.com/ a20v41n26/20412626.html

Ordóñez-Ortega, O., Gualdrón-Pinto, E., \& Amaya-Franky, G. (2019). Pensamiento variacional mediado con baldosas algebraicas y manipuladores virtuales. Revista de Investigación, Desarrollo e Innovación, 9(2), 347-362.

Orozco-Báez, M. Y., Niño-Vega, J. A., \& Fernández-Morales, F. H. (2020).
Estrategia pedagógica para formar a padres y acudientes sobre el fenómeno del ciberacoso. RevistaEspacios, 41(33), 24-33. https://www.revistaespacios. com/a20v41n33/20413303.html

Padilla-Ospina, A. M., Medina-Vásquez, J. E., \& Ospina-Holguín, J. H. (2020). Métodos de aprendizaje automático en los estudios prospectivos desde un ejemplo de la financiación de la innovación en Colombia. Revista de Investigación, Desarrollo e Innovación, 11(1).

Pineda-Ospina, D. L., Rodríguez-Guevara, E. G., \& García-Bonilla, D. A. (2020). Clústeres regionales como estrategia para superar desventajas competitivas. Revista de Investigación, Desarrollo e Innovación, 11(1).

Quitian-Feliciano, I. D., Rodríguez-González, G. L., \& Morales-Rubiano, M. E. (2020). Desafíos de los centros de gestión de la investigación para promover la investigación colaborativa. Revista de Investigación, Desarrollo e Innovación, 11(1).

Ruiz-Macías, E., \& Duarte, J. (2018). Diseño de un material didáctico computarizado para la enseñanza de Oscilaciones y Ondas, a partir del estilo de aprendizaje de los estudiantes. Revista de Investigación, Desarrollo e Innovación, 8(2), 295-309.

Suárez-Triana, Y., Rincón-Duran, R., \& Niño-Vega, J. (2020). Aplicación de herramientas web 3.0 para el desarrollo de competencias investigativas en estudiantes de educación media. Pensamiento Y Acción, (29), 3-20. https://revistas.uptc.edu.co/index.php/ pensamiento accion/article/view/11069 
Vargas-Vargas, N. A., Niño-Vega, J. A., \& Fernández-Morales, F. H. (2020). Aprendizaje basado en proyectos mediados por tic para superar dificultades en el aprendizaje de operaciones básicas matemáticas. Revista Boletín Redipe, 9(3), 167-180. https://doi.org/10.36260/rbr.v9i3.943

Vesga-Bravo, G. J., \& Escobar-Sánchez, R. E. (2018). Trabajo en solución de problemas matemáticos y su efecto sobre las creencias de estudiantes de básica secundaria. Revista de Investigación, Desarrollo e Innovación, 9(1), 103-114. 